\title{
Characterization and application of bioactive compounds in oil palm mesocarp fiber superheated steam condensate as an antifungal agent
}

\begin{abstract}
Lignocellulosic degradation products from superheated steam (SHS) pretreatment of oil palm mesocarp fiber (OPMF) at $190{ }^{\circ} \mathrm{C}$ to $240{ }^{\circ} \mathrm{C}$ for 1 hour were recovered as a condensate. Compositional analysis of the condensate was carried out and subsequently its potential application as an antifungal agent was investigated. GCMS analysis revealed a total of 62 compounds in the condensate sample from SHS pretreatment at $240{ }^{\circ} \mathrm{C}$, which were identified and classified into ten different groups of aromatic phenolics, furans, pyrans, dioxoles; and open chains ketones, esters, alcohols, aldehydes, alkenes and alkanes. The presence of carboxylic acids was identified by HPLC and the condensate contained acetic, formic, levulinic and succinic acids at concentrations of $1671 \mathrm{mg} \mathrm{L}^{-1}, 12320 \mathrm{mg} \mathrm{L}^{-1}, 831 \mathrm{mg}$ $\mathrm{L}^{-1}$ and $435 \mathrm{mg} \mathrm{L}^{-1}$, respectively. Complete suppression of Ganoderma boninense UPM13 mycelial growth was observed in the agar dilution test, while spore germination of Aspergillus fumigatus UPM2 and Trichoderma asperellum UPM1 was completely inhibited in the spore germination test. The growth suppression of $G$. boninense, a fungal causing basal stem rot (BSR) disease in oil palm plantation shows the condensate potential benefit to combat the disease.
\end{abstract}

Keyword: Oil Palm Mesocarp Fiber (OPMF); Superheated steam (SHS) pretreatment 